\title{
Health Facility Related Factors Influencing Utilization of the Partograph in Management of Women in Labor among Nurses in Machakos County
}

\author{
Mosoti Abuga T*, Kabue P and Keraka M \\ Department of Reproductive health, Kenyatta University, Kenya
}

*Corresponding author: Titus Mosoti Abuga, Department of Reproductive health, Kenyatta University, Kenya, Tel: 0721626033; Email: borayajoshua@gmail.com

\section{Research Article}

Volume 3 Issue 5

Received Date: September 26, 2019

Published Date: October 17, 2019

DOI: $10.23880 /$ nhij- 16000205

\section{Abstract}

A partogram is a graphical representation of the fetal status, maternal status and progress of labor. Its proper interpretation assists in early identification of complications which may lead to morbidity and mortality. Health records from selected hospitals in Machakos County in regards to partograph utilization revealed that $60 \%$ of the records had a partograph attached with only $40 \%$ of them used correctly up to delivery. The study determined the utilization of the partograph in management of women in labor in Machakos County. The objectives of the study were to determine the level of utilization of the partograph, and health facility related factors influencing the utilization of the partograph. A cross-sectional study design was used. The study was conducted in selected hospitals within Machakos County. The study population comprised of 124 nurses working in maternity wards. Purposive sampling technique was used to choose study participants from selected health facilities. Structured questionnaires were used to collect quantitative data while focused group discussions were conducted and key informants interviewed. Data collected was organized and entered into computer software and analyzed using SPSS Version 24 and presented by use of tables, figures, bar charts and narration. Logistic regression was used to assess the possible association and strength of association was measured using 95\% confidence interval. From the study, level of utilization of the partograph among midwives was at 44.5\%, nurses related factors influencing utilization of the partograph were negative attitude $(\mathrm{p}=0.001)$, and lack of training on partograph use $(\mathrm{p}=0.001)$. Majority $(55.5 \%)$ of the files reviewed were not completed while monitoring labor, Health related factors that were found to influence partograph use were number of deliveries in labor ward ( $p=0.001)$ whereby $53.6 \%$ of nurses conducted more than 5 deliveries per shift, and institutional policies on partograph use ( $\mathrm{p}=0.001$ ), in which $52.7 \%$ indicated that partographs were not available in labor wards while $68.2 \%$ said that institutional policies on partograph use were not implemented. The study concludes that utilization of partograph was poor, there was inadequate seminars, understaffing issues in labor wards and lack of policies on partograph use. The study recommends hospital administrators in the health facilities to arrange for obstetric care training for nurses on partograph utilization 


\section{Nursing \& Healthcare International Journal}

maternity in charges to ensure regular supply of partograph papers in labor wards, hospital managers to carry out regular supportive supervision in order to assess partograph utilization and make a proposal to the County ministry of health to employ more nurses towards improving utilization of partograph in Machakos County. Effective and efficient utilization of partograph helps curb maternal and fetal complications related to prolonged labour.

Keywords: Partograph use; Partograph utilization; Machakos county; Health related factors

\section{Introduction}

\section{Background of the Study}

The partogram which is also called a partograph is a graphical record used to monitor progress of women during labour. The partogram provides a pictorial overview of labour, alerts midwives and obstetricians to any deviations in maternal or fetal well-being and the progress of labour. It also assists in early decision making on transfer, augmentation or immediate delivery. The concept of a partogram was introduced by Friedman in 1954 by graphically plotting the dilation of the cervix against time during labour. Later after Friedman, Philpott and Castle in 1972 developed the partograph into a tool for monitoring labour by introduced the action and alert lines to the graphical record [1].

The World Health Organization (WHO) advocates the utilization of a partograph as the effective tool for monitoring labor and reducing labor complications in developing countries which is an important intervention in reducing both maternal and perinatal mortality and morbidity towards achieving the Millennium Development Goals 4 and 5 [2].

The different variables of a partogram includes: fetal condition which can be assessed through fetal heart rate, status of the membranes, quality of liquor and the degree of molding on the fetal head. The progress of labor records the rate of dilation of the cervix, strength and pattern of uterine contractions and the descent of the presenting part while maternal records includes maternal pulse rate, temperature, blood pressure and urine of the mother [3].

A study in western countries reported that there was no consensus over the utilization of the partograph which led variations in practice between the units. In the same study the heads of midwifery institutions explored the status of labor documentation and the results indicated that there was support for the utilization of the partograph although some facilities chose not to use it. Utilization of the partograph increases the analysis and skills of midwives in monitoring of pregnant women in labor which aids in providing standardized fetal and maternal care. The utilization of the partograph allows early recognition of obstructed labor and reducing chances of prolonged labor, postpartum hemorrhage and unnecessary caesarean section [1].

The shortage of midwives influences the quality of care offered to women in labor. Women in labor require close monitoring and recordings by the qualified midwife at a short interval during labor. Shortage of midwives hinders proper monitoring of mothers in labor [4]. In cases where there are so many clients, a midwife becomes exhausted which may lead to using short cuts leading to underutilization of the partograph [5].

Number of midwives who to attend to women in labor play a role in the utilization of the partograph. More staffs on duty utilize the partograph more than when there is a shortage of midwives attending to mothers in labor. On the same high number of partographs are completed when there are more staffs in maternity unit than when there were few midwives on duty to attend to women in labor [6].

A study by Survey (2015) [7] on utilization of partograph, midwives who were interviewed demonstrated that heavy workload in labor ward influenced the quality of care offered to women in labor. The study further revealed that since women in labor required close monitoring, heavy workload was likely to hinder proper monitoring of mothers in labor which led to underutilization of the partograph in monitoring women in labor. Similarly, findings by Harvey (2014) [8] showed that when nurses working in maternity wards cared for more women in labor in a given shift then they were likely to be fatigued as a result of heavy workload which compromised the care of mothers in labor. 


\section{Nursing \& Healthcare International Journal}

Data across African countries has shown that poor utilization of the partographs associated with lack of resources which include: pre-printed graph papers, surgical supplies and equipment such as blood pressure machines, thermometers, gloves and charts used by midwives in the labor wards [9]. Most of these supplies lack in most of the maternity units which made it difficult for midwives to monitor mothers in labor using the partograph [1].

A study finding by Abebe, et al. (2013) [10] on the impact of supervision on partograph use among nurses working in maternity ward, the study showed that supervision by head of departments in maternity wards influenced utilization of the partograph positively. Further, the study indicated that supervision ensured that nurses were able to remember to utilize the partograph while monitoring women in labor. In another study, partograph utilization in tertiary level hospital was promoted by frequent supervision by the departmental heads in labor ward [5].

Kenya is still one of those developing countries with high maternal mortality ratio and neonatal mortality rate, estimated at 510/100,000 [11] and 22/1000 live births [9]. Prolonged and obstructed labor accounts for $8-10 \%$ of maternal deaths while mechanical obstruction in second stage accounts for complications in about $1-2 \%$ of labor. Utilization of the partograph engender gross reduction in the number of deaths of both the mother and unborn baby by ensuring that complications are detected early for prompt interventions thereby enhancing maternal and neonatal well-being [4].

However, a study in Kenyatta National Hospital on partograph use revealed that partographs were incomplete, incorrectly filled or not used at all [12]. The use of partographs was introduced after some nurses had been trained and certified as qualified nurses. Therefore, not all nurses have been trained on partograph using. Health records from selected hospitals in Machakos County in regards to partograph utilization revealed that $60 \%$ of the records had a partograph attached with only $40 \%$ of them used correctly up to delivery. If nurses working in maternity wards used a partograph correctly in managing women in labor, there should be no complications which may lead to neonatal and maternal mortality. However, partograph use and documentation is a major problem in the majority of the health facilities which offer maternity services in Machakos County.

\section{Methodology}

A cross-sectional study was used to describe the utilization of the partograph by nurses in selected hospitals within Machakos County. This design was used because it provided relevant facts and opinions of individuals. The study was hospital based conducted in Machakos County, Kenya. The study targeted 120 nurses working in maternity departments within the selected hospitals in Machakos County, Kenya. The nurses participated either in structured questionnaire or focused group discussion but not both. The key informants included nurse in charges with midwifery background among the selected hospitals. The hospitals were divided into 3 stratus and proportionate sampling used to select respondents from each stratum. One level V Hospital and two-Level IV Hospitals from Machakos County were chosen for this study since they are the main hospitals which offer high volume maternity services. Purposive sampling technique was used to select nurses working in maternity since the study focused on particular characteristics of a population of interest which enabled to answer the research objectives.

In focus group discussion, maternity nurses who had not participated in structured questionnaire were selected. The sampled nurses participated in FGD using FGD guide on partograph utilization. Key informants were selected purposively from the maternity in charges because they had firsthand knowledge on partograph use in labor wards. A pretest was carried out to determine reliability of the questionnaires. Reliability analysis was subsequently done using Cronbach's Alpha which measured the internal consistency by establishing if certain item within a scale measures the same construct.

Data was collected using structured interviews which were carried out using a questionnaire to afford quantitative analysis. The questionnaire featured both closed and open-ended questions. A combination of both qualitative and quantitative questions was used to collect data. To ensure validity, the questionnaire was tested, issues which were not clear were clarified, all questions were thoroughly scrutinized by technical persons and those which were not necessary were deleted. Those questions that needed to be rephrased were edited accordingly before the study was commenced. Content validity was ensured through thorough proof reading and peer review of the tool before it was subjected to the respondents. Continuous supervision from the supervisors added value to the validity of the tool. This was assured through counter checking the filled 


\section{Nursing \& Healthcare International Journal}

questionnaires on a daily basis in order to identify and correct any error that might have occurred which enhanced accuracy and consistence of the tool. After coding of the results in SPSS, reliability was tested and found Cronbach's alpha to be at 0.835 (Table 1).

\begin{tabular}{|c|c|c|}
\hline Scale & $\begin{array}{c}\text { Cronbach's } \\
\text { Alpha }\end{array}$ & $\begin{array}{c}\text { Number of } \\
\text { Items }\end{array}$ \\
\hline $\begin{array}{c}\text { Health facility related factors } \\
\text { influencing partograph } \\
\text { utilization }\end{array}$ & 0.835 & 5 \\
\hline
\end{tabular}

Table 1: Reliability analysis table.

This was evident enough that the tool was reliable. Data collected was cleaned, edited, coded and analyzed using the Statistical Package for the Social Sciences (SPSS) software, version 24. Data was then summarized using descriptive statistics such as frequencies and presented by use of tables, figures, and narration. Analysis of contingency tables was done to establish relationship between variables and Chi Square was used to test the relationship between variables. Results from FGDs and KIIs were arranged in general categories identified in the discussion guidelines then coded, common themes were identified, inferences made from each theme and conclusions drawn during thematic analysis.

Approval to conduct the study was given by Kenyatta University Ethics and Review Committee and the authority to undertake the study was sought from the National Commission for Science, Technology and Innovation. Permission to carry out research was sought from County Director of Health services, Medical superintendent from each selected Hospitals were informed. The nursing officers in charges of the selected hospitals were informed. The questionnaires were administered to the respondents upon obtaining an informed consent. To ensure privacy, names and other means of identity were not used during the data collection. The researcher ensured that all information obtained was kept under lock and key and was only used for the purpose of the study.

\section{Utilization Measurement}

A retrospective spot check was done on the files of mothers who had already delivered within one week before the study. The files used to assess utilization had a partograph attached to it. The level of utilization was measured using a utilization level scale where a score of 1 was awarded for those files which were dully filled and completed while a score of 0 was given to those files which were not dully completed or not filled at all. The number of files picked from each facility were randomly picked then evaluated for level of partograph utilization. The total number of files picked from each facility was the same to that of the sample size. This promoted representativeness and validity of the information on partograph utilization among nurses from the selected health facilities.

\section{Response Rate}

The study targeted a sample size of 124 nurses working in maternity departments from which 110 filled in and returned the questionnaires making a response rate of $88.7 \%$. This response rate was satisfactory to make conclusions for the study as it acted as a representative. According to Mugenda, et al. (2003) [13], a response rate of $50 \%$ is adequate for analysis and reporting; a rate of $60 \%$ is good and a response rate of $70 \%$ and over is excellent. Based on the assertion, the response rate was excellent.

\section{Results}

\section{Demographic Characteristics of the Participants}

The study involved nurses who had worked in labor ward for at least six months. The study established that more than half of the respondents 78 (70.9\%) were female. The mean age of the respondents was calculated to be $26.64 \pm 2.6$ years and nearly half of them $58(52.7 \%)$ were within the age group of 25-30 years. More than half $58(52.7 \%)$ of the respondents were from Machakos level 5 while the least $22(20 \%)$ were from Kathiani level 4 hospital. A considerable number of respondents, 91 $(82.5 \%)$ were diploma holders in Kenya Registered community health nursing, $11(10 \%)$ had a diploma in midwifery and $8(7.5 \%)$ had a degree in nursing, the remaining respondents had a certificate in nursing. Majority of the nurses 93 (84.5\%) had worked in labor ward for less than six years.

The study involved nurses who had worked in labor ward for at least six months. The study established that majority of the respondents were female with the mean age of 27 years and nearly more than half of them were within the age group of 25-30 years. In addition, more than half of the respondents were from Machakos level 5 hospitals with the least of the respondents being from Kathiani Level 4 hospital. On qualifications of the respondents, majority of respondents had diploma in Kenya Registered community health nursing. However, 
the minority had a diploma in midwifery while the least had a degree in nursing. In addition, majority of nurses had worked in labor ward for less than six years.

\begin{tabular}{|c|c|c|}
\hline Variables & Frequency $(\mathrm{N}=110)$ & Percentage \\
\hline \multicolumn{3}{|c|}{ Health institution } \\
\hline Machakos level 5 hospital & 58 & 52.7 \\
\hline Kangundo level 4 hospital & 30 & 27.3 \\
\hline Kathiani level 4 hospital & 22 & 20 \\
\hline \multicolumn{3}{|c|}{ Gender } \\
\hline Male & 32 & 29.1 \\
\hline Female & 78 & 70.9 \\
\hline \multicolumn{3}{|c|}{ Qualification Level } \\
\hline Certificate & 9 & 8.2 \\
\hline Diploma & 93 & 84.5 \\
\hline Degree & 8 & 7.3 \\
\hline \multicolumn{3}{|c|}{ Years of experience in labor ward } \\
\hline 6 Months $\leq 3$ years & 27 & 24.5 \\
\hline 3-6 years & 66 & 60 \\
\hline$\geq 6$ years & 17 & 15.5 \\
\hline \multicolumn{3}{|c|}{ The profession of the respondent } \\
\hline KRCHN & 91 & 82.5 \\
\hline Midwifery & 11 & 10 \\
\hline Degree in nursing & 8 & 7.5 \\
\hline
\end{tabular}

Table 2: Demographic characteristics of the participants.

\section{Level of Utilization of Partograph Charts among Maternity Nurses in Machakos County}

The results of study revealed that majority of the nurses, $61(55.5 \%)$ did not duly complete the partograph charts available in labor ward. This was attributed to a number of factors ranging from nurses related factors and health institution related factors from where they were working (Table 3).

\begin{tabular}{|c|c|c|c|}
\hline Variable & Category & Frequency & Percentage \\
\hline $\begin{array}{c}\text { General utilization of the partographs in labor } \\
\text { ward from selected hospitals }\end{array}$ & Completed partographs & 49 & $44.50 \%$ \\
\cline { 2 - 4 } & Non completed partographs & 61 & $55.50 \%$ \\
\hline
\end{tabular}

Table 3: Utilization of the partograph among maternity nurses in Machakos County.

Utilization of the partograph was assessed by looking at the files retrogressively on the completion of the partographs during monitoring labor. General utilization of the partographs in labor ward among nurses from selected hospitals revealed that majority of the files were not fully completed (started to be filled but not completed) while monitoring women in labor. Several similar studies confirmed the low utilization of the partograph in Africa [14]. This finding is lower than studies conducted in Nigeria (98.8\%) but higher than the findings from other studies like; Addis Ababa, Ethiopia $(57.4 \%)$. The possible reason for this variation could be due to the availability of well-designed and coordinated programs like; the strength of mentorship, supportive supervision [15]. Moreover, inadequate utilization of the partograph could be part of the reasons for high maternal mortality in developing countries [4]. In the current study therefore, this necessitates the need for regular on job training among midwives on partograph use for safety of women in labor.

Utilization of partogram requires skills as part of the Safe Motherhood Initiative. The WHO's partograph clearly differentiates normal from abnormal progress of labour and identifies those women likely to require intervention. Its use in all labour wards is recommended [16]. However, despite majority of the nurses having the required knowledge and skills necessary for partograph use, other 


\section{Nursing \& Healthcare International Journal}

factors influencing its utilization can be negative attitude and workload in health institutions where mothers are admitted for delivery [2].

\section{Health Facility Related Factors Influencing Utilization of the Partograph among Maternity Nurses}

Various aspects of the health facility were assessed, specifically on; number of deliveries per shift, staffing, availability of the partograph charts, use of available charts, institutional policies on partographing, and availability of resources for monitoring a mother in labor.

During the study, the respondents were asked to report the ratio of nurses to mothers in labor per that shift. On the ratio of nurses to mothers in labor, 52 $(47.3 \%)$ indicated that one nurse monitored more than 5 mothers in labor, $40(36.4 \%)$ one monitored 5 mothers in labor while $18(16.4 \%)$ indicated one nurse to one mother. In addition, 59 (53.6\%) indicated that they had 5 to 10 deliveries per shift, 41 (37.3\%) more than 10 deliveries while $10(9.1 \%)$ one to 5 deliveries per shift.

Whether the increase in the number of deliveries led to increase in the number of staffs (nurses) in the unit, $86 \%(78.2 \%)$ indicated that there was no increase in the number of staffs. More than 58 (52.7\%) indicated that partographs were not available in the unit. Moreover, on the use of available partographs, $61(55.5 \%)$ agreed that they were used. The majority of the respondents 75 $(68.2 \%)$ indicated that their hospitals had policies on partograph use. However, they were not followed while monitoring women in labor. In addition, 85 (77.3\%) revealed that resources used to monitor mothers in labor were available.

\section{Ratio of Mother in Labor to Number of Maternity Nurses per Shift}

The number of nurses who were working per shift varied from one hospital to another. For those who were in a ratio of one nurse to five mothers were 40 (36.4\%), those with a ratio of one nurse to more than five mothers in labor were represented by $52(47.3 \%)$ while the remaining $18(16.4 \%)$, represented cases where the ratio of nurses to mothers in labor was one nurse to two mothers in labor. However, this was not significantly associated with utilization of partograph $\left(\chi^{2}=1.652\right.$, $\mathrm{p}=0.438$ ). Number of midwives who to attend to women in labor play a role in the utilization of the partograph. However, in the current study majority of the midwives cared for more than 5 mothers in labor in a given shift.
This is inconsistent with findings by Opoku, et al. (2015) [17] where adequate number of nurses in labor ward utilized the partograph more than when there is a shortage of midwives attending to mothers in labor. Furthermore, the findings of this study reveal shortage of staff per shift in labour ward where one midwife cared for more than 5 mothers in labor per shift which led to poor utilization of the partograph.

\section{Number of Deliveries per Shift versus the Number of Maternity Nurses in Machakos County}

In most of the hospitals, they reported increased number of deliveries while the number of staffs per department remained constant. Those hospitals which a range of deliveries between one and five deliveries, the deliveries accounted for 10 (9.1\%), those that had five to ten deliveries per shift were 59 (53.6\%) while those who had more than ten deliveries per shift were represented by $41(37.3 \%)$. There was a significant relationship between the number of deliveries and the number of nurses per shift $\left(\chi^{2}=1.714, p=0.002\right)$. Focused group discussion revealed that "during peak season, there are more deliveries than the nurses can handle which lead to inadequate utilization of the partograph" (FGD nurse in Kangundo). In another FGD, "this being a referral hospital, maternity ward is always full and the number of nurses in the unit is constant despite the increased workload which prevents utilization of the partograph" (FGD nurse in Machakos level 5 hospital). In Machakos, focused group discussion revealed that "adequate staffing of nurses in labor ward can ensure successful utilization of partograph in management of women in labor" (FGD nurses in Machakos level 5 hospital).

Moreover, challenges influencing utilization of the partograph was cited by the informers, "lack of adequate resources which are sustainable remains a challenge towards utilization of the partograph" (key informer, Kangundo level 4 hospital). This was different with other findings where informer revealed, "understaffing in labor wards remains a challenge because one nurse monitored more than 5 mothers in labor which influenced utilization of partograph" (FGD nurse in Machakos level 5 hospital).

The increased in number of deliveries per shift led to low utilization of partographs in maternity wards. This was revealed by $86(78.2 \%)$ of the respondents reporting that increase of deliveries led to low use of partographs. Increase in number of deliveries with a constant number of staffs significantly affected use of partographs 


\section{Nursing \& Healthcare International Journal}

$\left(X^{2}=10.273, P=0.001\right)$. Findings from the key informants revealed that inadequate staffing and lack of frequent supervision were among the health facility factors influencing utilization of the partograph, "when there are no Kenya medical students (KMTC) who help us in maternity, the available nurses are overwhelmed with the number of mothers in labor which contributes to nonutilization of the partograph" (key informer from Machakos level 5 hospital). In addition, "some nurse in charges do not carry regular supervision on partograph use among nurses working in maternity wards, this leads to laxity among nurses which lead to underutilization of the partograph" (key informer from Kangundo level 4 hospital).

\section{Availability of Partographs in Labor Ward and its Use by Maternity Nurses}

Majority of the respondents $58(52.7 \%)$ indicated that partograph papers were not available in labor wards while 52(47.3\%) reported that the partographs were available in labor wards. Moreover, on further probing, the available partographs were not all duly completed. More than half of the partographs 61 (55.5\%) were duly documented while the remaining were half way completed. When the researcher probed further on use of available partograph charts, it was evident that the nurses used the available partograph charts. Statistically, availability of partograph papers contributed to utilization of the partograph $\left(\chi^{2}=4.583, \mathrm{P}=0.001\right)$. Lack of partograph chart papers featured where, "sometimes we do not have regular supply of partograph papers and therefore we are forced to document the findings on the cardex" (FGD nurse from Kathiani level 4 hospital).

In addition, nurses from Kathiani revealed that lack of regular supply of partograph papers led to inadequate utilization of the partograph "the photocopy machine sometimes developed mechanical problems which leads to inadequate supply of partograph papers, it can take up to 6 months before it becomes operational which influences utilization of partograph" (FGD nurse from Kathiani level 4 hospital).

Availability of essential resources such as the partograph papers was key in management of labor. However, partograph papers were not available in the majority of labor wards. In agreement, Khonje (2014) [18] indicated that majority of the participants in the study area reported unavailability of partogram papers in labor wards which hindered utilization of partograph. However, despite the partograph papers unavailable in the majority of health institutions, the few available partographs were not completed in management of labor including in the current study.

\section{Availability of Institutional Policies and its Effect on Partograph Use by Maternity Nurses}

Most of the respondents 78 (70.9\%) reported that the hospital had laid down policies on use of partographs. On assessment of the use of the policies in relation to duly filling of the partographs, the respondents reported that in most cases $75(68.2 \%)$ the policies were not put into practice. On the availability of institutional policies and partograph use, the findings were statistically significant $\left(\chi^{2}=14.457, \mathrm{P}=0.001\right)$. The policies were available but not implemented. Availability of institutional policies was statistically significant towards utilization of partograph. Despite majority of the respondents indicating that their facilities had laid down policies on partograph use, policies on partograph use were not implemented in utilization of the partograph. This contrasts with the findings by Shazly, et al. (2014) [19] where majority of nurses interviewed from different health facilities in South Africa did not have institutional policies on partograph use. However, partograph utilization was noted in the majority of the health institutions despite lacking institutional policies on partograph use. Inadequate use of institutional policies on partograph use can be attributed to negative attitude among midwives which influences partograph use.

\section{Availability of Resources for Monitoring Partograph Regularly among Maternity Nurses}

In most of the hospitals, the respondents reported that the equipment for monitoring the mothers in labor were available. On assessment of the available equipment in labor,85 (77.3\%) of the respondents from the hospitals had all the equipment necessary for monitoring a mother in labor; however, it was evident that the nurses in those hospitals were using the equipment to monitor mothers in labor but didn't document the findings on partographs charts $\left(\chi^{2}=0.271, P=0.603\right)$.

Majority of the respondents reported that the equipment for monitoring mothers in labor were available. However, it was evident that the nurses were using the equipment to monitor mothers in labor but they did not document the findings on partographs charts. This is different with the findings by Harvey (2014) [8] where majority of nurses interviewed lacked most of the supplies in the maternity units which made it difficult for midwives to monitor mothers in labor using the 
partograph. From the study findings, inadequate partograph utilization despite having necessary resources could be attributed to other factors such as understaffing in labor ward and negative attitude among nurses caring for women in labor. On what can be improved in the institutions to ensure successful utilization of the partograph, provision of resources, adequate staffing and regular and continuous supervision was key towards ensuring successful partograph use "if the hospital provided regular supply of resources such as partograph charts then utilization of partograph can be improved" (FGD nurse in Kathiani level 4 hospital). In Kangundo level 4, "regular and continuous supervision among nurses can lead to improved utilization of the partograph" (FGD from Kangundo level 4 hospital).
In order to ensure successful utilization of the partograph, majority of the nurses reported that provision of resources, adequate staffing and regular continuous supervision was key towards ensuring successful partograph use in management of women in labor. This concurs with the findings by Sinha, et al. (2017) [20] in South Africa which indicated that nurses need to be supported through provision of resources both human and material in order to promote adequate partograph use among nurses working in labor ward. In addition, Harvey (2014) [8] indicated that support in terms of provision of resources such as the partograph itself and equipment required for completion of the partograph is vital at a basic level to ensure consistent use of the partograph in the management of women in labor (Table 4).

\begin{tabular}{|c|c|c|c|c|c|}
\hline \multirow[b]{2}{*}{ Variable } & \multirow[b]{2}{*}{ Category } & \multicolumn{2}{|c|}{ Use of partograph $(\mathrm{N}=110)$} & \multirow[b]{2}{*}{$P$ df $\chi^{2}$} & \multirow[b]{2}{*}{$\begin{array}{l}\text { Likelihood } \\
\text { Ratio }\end{array}$} \\
\hline & & $\begin{array}{l}\text { Properly } \\
\text { used }\end{array}$ & $\begin{array}{l}\text { Not properly } \\
\text { used }\end{array}$ & & \\
\hline \multirow{3}{*}{ Deliveries per shift } & 01-May & $1(0.9 \%)$ & $9(8 \%)$ & \multirow{3}{*}{$\begin{array}{c}\mathrm{P}=0.002 \mathrm{df}=2 \\
\chi 2=12.440\end{array}$} & \multirow{3}{*}{$\mathrm{OR}=1.737$} \\
\hline & 05 -Oct & $31(28.1 \%)$ & $28(25.5 \%)$ & & \\
\hline & $>10$ & $29(26.3 \%)$ & $12(11 \%)$ & & \\
\hline \multirow{2}{*}{$\begin{array}{l}\text { Effect of increased deliveries on } \\
\text { partograph use }\end{array}$} & $\begin{array}{c}\text { High } \\
\text { utilization }\end{array}$ & $20(18.1 \%)$ & $9(8 \%)$ & \multirow{2}{*}{$\begin{array}{c}P=0.001 \mathrm{df}=2 \\
\chi 2=10.273\end{array}$} & \multirow{2}{*}{$\mathrm{OR}=3.311$} \\
\hline & $\begin{array}{c}\text { Low } \\
\text { utilization }\end{array}$ & $28(25 \%)$ & $53(48.1 \%)$ & & \\
\hline \multirow{2}{*}{$\begin{array}{c}\text { Availability of partograph charts in labor } \\
\text { ward }\end{array}$} & Available & $45(40.9 \%)$ & $7(6.6 \%)$ & \multirow{2}{*}{$\begin{array}{c}P=0.001 \mathrm{df}=1 \\
\chi 2=4.583\end{array}$} & \multirow{2}{*}{$\mathrm{OR}=6.722$} \\
\hline & Not available & $48(43.6 \%)$ & $10(9 \%)$ & & \\
\hline \multirow{2}{*}{$\begin{array}{c}\text { Are institutional policies on partograph } \\
\text { implemented }\end{array}$} & yes & $26(24 \%)$ & $9(8 \%)$ & \multirow{2}{*}{$\begin{array}{c}\mathrm{P}=0.001 \mathrm{df}=1 \\
\chi 2=7.369\end{array}$} & \multirow{2}{*}{$\mathrm{OR}=7.776$} \\
\hline & No & $35(31.8 \%)$ & $40(36.4 \%)$ & & \\
\hline
\end{tabular}

Table 4: Summary of health related factors affecting utilization of partograph.

\section{Conclusion of the Study}

Utilization of the partograph among nurses working in labor department was poor. Health facility related factors influencing utilization of the partograph were understaffing, unavailability of partograph papers and lack of implementing policies on partograph use. Nurses working in labor ward cared for at least 5 mothers in labor, labor wards lacked regular supply of partograph papers while maternity units did not implement policies on partograph use.

\section{Recommendations of the Study}

Maternity in charges should work closely with health administrators to ensure regular supply of partograph papers in labor wards and timely procurement of materials necessary in labor wards in order to improve utilization of partograph in management of women in labor. The hospital managers should make a proposal to the County policy makers in the ministry of health on the need to employ more nurses in order to address the issue of understaffing in labor wards towards improving utilization of partograph in management of women in labor in Machakos County.

\section{Acknowledgement}

I thank the almighty God for his grace, strength, guidance, good health and sustenance throughout the courses of study. I acknowledge Dr.Kabue and Professor Keraka for their tireless efforts in guiding me through the process of writing this thesis. I am also very grateful to nurses working in Machakos level 5, Kangundo and Kathiani level 4 hospitals and the in charges for their assistance during data collection. Not forgetting Mr. 


\section{Nursing \& Healthcare International Journal}

Boraya for his great contribution in data analysis. God bless you all.

Conflict of Interest: None declared.

\section{References}

1. Lavender T, Hart A, Smyth RM (2013) Effect of partogram use on outcomes for women in spontaneous labour at term. Cochrane Database Syst Rev (7): CD005461.

2. Soni BL (2014) Effect of partogram use on outcomes for women in spontaneous labour at term: RHL commentary, The WHO Reproductive Health Library, World Health Organization, Geneva.

3. Magon N (2013) Partograph Revisited. International Journal of Clinical Cases Investigations 3: 1-6.

4. WHO (2013) Trends in maternal mortality: 19902013, Estimates by WHO, UNICEF, UNFPA, the World Bank and the United Nations population, World Health Organization, Geneva: Switzerland.

5. Fawole AO, Hunyinbo KI, Adekanle DA (2013) Knowledge and utilization of the partograph among obstetric caregivers in South West Nigeria. Afr J Reprod Health 12(1): 46-72.

6. Opiah MM, Ofi AB, Essien EJ, Monjok E (2012) Knowledge and utilization of the partograph among midwives in the Niger Delta Region of Nigeria. Afr J Reprod Health 16(1): 125-132.

7. Survey DK (2015) Maternal and child survival. Kenya: Management of women in labor. Kenya Bureau of Statistics.

8. Harvey SA (2014) Skilled Birth attendance competence and its implication in Safe Motherhood movement. International Journal for Obstetrics and Gynecology 87: 203-210.

9. Kenya Demographic and Health Survey (KDHS) (2014-2015). Count down to 2015 Maternal and Child Survival, Kenya.

10. Abebe F, Birhanu D, Awoke W, Eligu T (2013) Assessment of knowledge and utilization of the partograph among Health professionals in Amhara region. Science journal of clinical medicine 2(2).
11. World Health Organization (2015) World Health Organization's partograph in management of labour. Maternal Health and Safe Motherhood.

12. Rotich E, Maina L, Njihia A, Christensson K (2011) Evaluating partograph use at two main referral hospitals in Kenya. African Journal of Midwifery and Women's Health 5(1): 21-24.

13. Mugenda, Mugenda (2003) Research methodology textbook.

14. Wakgari N, Amano A, Berta M, Tessema A (2015) Partograph utilization and associated factors among obstetric care providers in North Shoa Zone, Central Ethiopia: a cross sectional study. Afr Health Sci 15(2): 552-559.

15. Bekele D, Beyene K, Hinkosa L, Shemsu N (2017) Partograph utilization and associated factors among graduating health professional students in Asella Referral and Teaching Hospital, Ethiopia. Journal of Computational Biology 6(2): 12-18.

16. Bedwell C, Levin K, Pett C, Lavender T (2017) A realist review of the partograph: when and how does it work for labour monitoring? BMC Pregnancy and Childbirth 17(1): 31.

17. Opoku BK, Nguah SB (2015) Utilization of the modified WHO partograph in assessing the progress of labour in a metropolitan area in Ghana. Journal for Women's Health 2(2): 1-7.

18. Khonje M (2014) A cross sectional study on use and documentation of partograph and factors that prevent optimal utilization of the partograph: perspective of health workers in maternity units in Lilongwe Malawi, University of Oslo, Norway 1: 1155.

19. Shazly SA, Embaby LH, Ali SS (2014) The labour scale assessment of the validity of a novel labour chart: A pilot study. Aust N Z J Obstet Gynaecol 54(4): 322326.

20. Sinha D, Shrivastava S, Shrivastava S (2017) Comparative study of 4-hour versus 2-hour action line on WHO modified partograph. International Journal of Research in Medical Sciences 5(3): 876879.

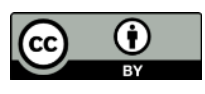

